easterly current over the N.W. monsoon and of an upper current over the S.E. trade, more southerly than the surface wind, is not only altogether new, but also quite anomalous.

In Australia, and the Southern Hemisphere generally, the upper current over a N.W. wind is from about W. and over a $\mathrm{S}$.E. wind from about $\mathrm{E}$.

On my way home I ran a section across the Atlantic from Rio to Teneriffe, but the absence of cirrus prevented any satisfactory determination of the upper winds in that region.

The matter is, however, so important that I start again in a few days for the hurricane region of Mauritius, where I hope to observe one of these exceptional cyclones. Then I hope to repeat a section of the Indian Ocean between Mauritius and Bombay, and afterwards, if all goes well, to get some sections in the Pacific to see what the meaning of this curious discovery may be.

2I, Chapel Street, S.W., October 26

\section{The Hellgate Explosion and Rackarock}

The statement in NATURE of the I5th inst. (p. 575) that rackarock is "blasting gelatine" or "nitroglycerine with compressed gun-cotton" is incorrect. Rackarock is simpiy powdered potassium chlorate, impregnated with an inexpensive oily combustible, such as coal-tar oil, and is one of my safetyexplosives, which I discovered in 1870 , patented in England, April 6 and October 5, 1871, and described more fully in the Fournal of the Chemical Society for August, 1873, under the title: "On a New Class of Explosives, which are non-Explosive during their Manufacture, Storage, and Transport."

I am not responsible for the quaint name which the Americans have been pleased to give to my child.

As the so-called " rackarock" is not very sensitive or easy to explode, it requires a strong primer or detonator to set it off. This property, which I have fully discussed and particularly accentuated in my paper of 1873 , explains why Gen. Newton, the Chief Engineer of the Hellgate mine, took the precaution of placing as a primer such a powerful charge ( 33 tons) of expensive dynamite on the cheaper charge of the potassium chlorate mixture ( 107 tons), a precaution carried here perhaps a little too far.

Still it is satisfactory to see that my safety-explosive performed the main part of the labour and rendered good service in the advancement of the works of peace.

Savile Club, 107, Piccadilly

[We are very pleased to insert Dr. Sprengel's correction as to the composition of "rackarock." Up to the time of our notice about the explosion going to press the only information we could obtain was that it was the same substance as blasting gelatine, but with a less portentous name.-ED.]

\section{An Earthquake Invention}

IN your number for October I5 (p. 573) your numerous scientific readers will be interested to find a pretty long letter under the above heading from so able a seismologist as Prof. John Milne, of Tokio, Japan. Yet, his invitation notwithstanding, I must decline any discussion with him, either about my old letters which he refers to, or his own much changed opinion on their subject, since the occasion for my writing them occurred.

Those points, Mr. D. A. Stevenson, who is also invited, may, or may not, take up. My letters were impersonal, and dealt only with a British Association Report. I desire also to continue to keep them strictly to that, even to the very words of the particular Report as given forth to the world with all the usually unquestioned authority of that mighty Association, in their B.A. volume for 1884 , p. 248 , Section entitled "Experiments on a Building to resist Earthquake Motion."

15, Royal Terrace, Edinburgh, October I6

\section{Piazzi Smyth}

\section{On the Behaviour of Stretched Indiarubber when} Heated

SOME time ago (NATURE, vol. xxv. p. 507) you permitted me to express a doubt as to the invariable success of an often-quoted experiment with cylinders of bismuth and iron, intended to llustrate some relations between specific heat and thermal conductivity. ${ }^{1}$ I regret that $I$ have made further progress along

I Perhaps I may mention in passing that if lead is substituted for the bismuth the experiment succeeds perfectly, as theoretically it should do. the evil road of scepticism. I should like, in fact, to ask whether it is absolutely true to say without qualification, as is done in many text-books, that india-rubber (when stretched) forms an exception to the general law that the volume of a body is increased when the temperature is increased. The usual form of the experiment supposed to prove this is well known : a piece of india-rubber tube or cord is stretched by a weight connected with a long light index-lever, and it is shown that when heat is applied the india-rubber gets decidedly shorter.

I have always had some hesitation in showing and explaining the result of the experiment in the above way, especially as I could not find any proof given that the contraction in length was not compensated, or more than compensated, by an expansion in other directions (like that of a worm in its creeping progress, or of a dry rope when wetted). I had, in fact, lately arranged an apparatus for determining the coefficient of expansion of indiarubber, whether positive or negative, when I found that the subject has been very fully investigated by Dr. J. Russner, of Chemnitz (see Carl's Repertorium for 1882, pp. 161 and 196).

His results are briefly these:-

(I) That india-rubber (of which several kinds were examined) has without exception a definite coefficient of expansion which is always positive; experiments made at temperatures varying from $0^{\circ}$ to $53^{\circ} 4$ gave, for its value at $10^{\circ}, 0^{\circ} 000657$; at $30^{\circ}$, 0.000670.

(2) That india-rubber in a stretched state expands to the same extent as when it is not stretched. No point of minimum density was observed, such as Puschi supposed to exist.

(3) That the apparently anomalous behaviour of stretched india-rubber when heated is simply a case analogous to those of anisotropic crystals, which expand to different extents in different directions. Iceland spar, for instance, as Mitscherlich showed, actually contracts in a direction at right angles to its principal axis when heated, although its volume is, on the whole, increased.

Although ordinary india-rubber is, of course, isotropic, yet when stretched it becomes anisotropic, as may easily be shown by stretching a piece until it is semi-transparent, and placing it between crossed Nicols; the direction of the strain lying at an angle of $45^{\circ}$ with the plane of polarisation. Distinct colours, as with a selenite film, will be seen, varying from red to blue with the amount of strain.

The fact that india-rubber becomes hot when stretched, and especially if stretched and allowed to contract several times in succession, may perhaps be accounted for by molecular friction. It would almost seem, then, that in the account given in many books the truth, as well as the india-rubber, has been slightly "stretched."

Eton College, October 23 H. G. MADAN

\section{The Resting Position of Oysters}

IN carrying out a series of experiments on the artificial breeding of oysters in my private aquaria, I noticed that the young oysters born in the tanks rested on the flatter shell when they obtained a flat surface, such as a tile, to adhere to, but when I so arranged that they had irregular surfaces to deal with, such as little bundles of twigs, some adhered one way, and some the other. But where young oysters, nearly two years old, were moved from their original supports, and were compelled to find new ones, they selected the flat shell to rest upon in every instance, except where they were placed on sand, in which case they rested on the convex shell, in order apparently to avoid clogging the mouth of the shell with sand. Is it not possible from these observations that adult oysters vary their position according to the nature of the ground they are on. I have seen adult oysters on muddy ground lying on the convex shell, while whete adhesion to a flat surface could be obtained, they were all on the flat shell, and pectens are dredged with Balari and other growths on the flat shell in some instances, and on the convex shell in others, principally, however, on the latter.

South Kensington Museum, October 23 H. Stuart-WortLey

\section{The Value of the Testimony to the Aurora-Sound}

I HAVE read with much interest the descriptions of this sound as given by Dr. Sophus Tromholt's correspondents in NATURE of September 24. I was, however, struck by the similarity of these descriptions to the well-known phenomena of tinnitus 